\title{
Case Report \\ Successful Bridge-to-Recovery Treatment in a Young Patient with Fulminant Eosinophilic Myocarditis: Roles of a Percutaneous Ventricular Assist Device and Endomyocardial Biopsy
}

\author{
Saki Hasegawa-Tamba, ${ }_{1}$ Keiki Sugi, ${ }^{1}$ Yodo Gatate, ${ }^{1}$ Kanako Sugiyama, \\ Toshihiro Muramatsu, ${ }^{1}$ Shigeyuki Nishimura, ${ }^{1}$ Masanori Yasuda, ${ }^{2}$ \\ Kenji Fukushima, ${ }^{3}$ and Shintaro Nakano ${ }^{1}{ }^{1}$ \\ ${ }^{1}$ Department of Cardiology, International Medical Center, Saitama Medical University, Saitama, Japan \\ ${ }^{2}$ Division of Pathology, Saitama Medical University, Saitama, Japan \\ ${ }^{3}$ Department of Nuclear Medicine, International Medical Center, Saitama Medical University, Saitama, Japan \\ Correspondence should be addressed to Shintaro Nakano; snakano@saitama-med.ac.jp
}

Received 18 February 2019; Revised 31 May 2019; Accepted 16 June 2019; Published 2 July 2019

Academic Editor: Vasileios Papadopoulos

Copyright (C) 2019 Saki Hasegawa-Tamba et al. This is an open access article distributed under the Creative Commons Attribution License, which permits unrestricted use, distribution, and reproduction in any medium, provided the original work is properly cited.

Eosinophilic myocarditis (EM) is a rare condition characterized by myocardial eosinophilic infiltration due to various underlying etiologies. The patient with EM may benefit from appropriate use of mechanical circulatory support (MCS) that acts as a bridge to myocardial recovery in response to effective immunosuppressive therapy. A 16-year-old boy presented with cardiogenic shock due to fulminant myocarditis, for which a percutaneous ventricular assist device (PVAD) was immediately inserted. Based on the histological diagnosis of EM, immunosuppressive therapy was immediately commenced, leading to improvement of left-ventricular ejection fraction (27\% to $47 \%)$. The PVAD was successfully removed on day 7. Cardiac magnetic resonance imaging and dualtracer myocardial scintigraphy suggested limited extent of irreversible myocardial damage. For fulminant EM, the short-term use of PVAD, together with immunosuppressive therapy guided by an immediate histological investigation, may be an effective bridging strategy to myocardial recovery.

\section{Introduction}

Eosinophilic myocarditis (EM) is a rare condition characterized by myocardial eosinophilic infiltration due to various underlying etiologies [1-4]. Although EM manifests as fulminant myocarditis that is often fatal $[5,6]$, the patient may benefit from appropriate use of mechanical circulatory support (MCS) that acts as a bridge to myocardial recovery in response to effective immunosuppressive therapy. Veno-arterial extracorporeal membrane oxygenation (VA-ECMO) is an established therapeutic MCS option for patients in severe cardiogenic shock due to fulminant myocarditis because it facilitates rapid induction of stable hemodynamics via a femoral approach [7-10]. Despite VAECMO, however, the mortality rate associated with cardiogenic shock is still high [11], presumably because of its unfavorable effects on hemodynamics such as an increase in the afterload. The Impella 2.5 system (Abiomed, Danvers, MA, USA), a miniature percutaneous left-ventricular (LV) assist device (PVAD), was launched in our county in September 2017. It equips axial-flow pumps from the left ventricle to the ascending aorta to unload the left ventricle as well as provide flow support up to $2.5 \mathrm{~L} / \mathrm{min}$ $[8,12]$.

We present the case of a young boy who survived fulminant EM with successful short-term MCS using PVAD 


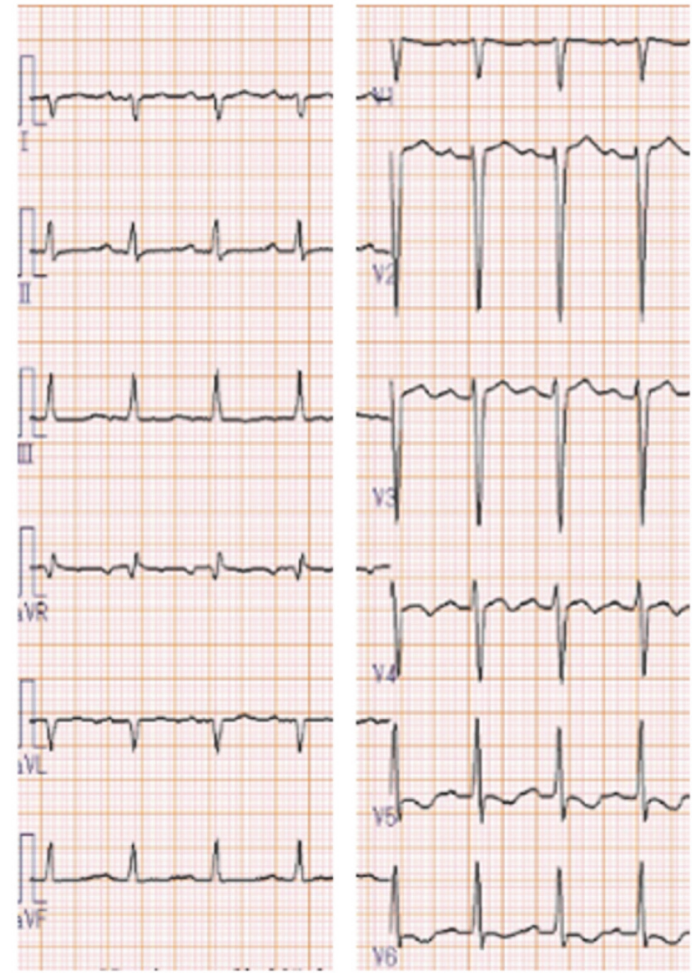

FIGURE 1: Electrocardiogram on admission. Electrocardiogram shows tachycardia, sinus rhythm, right axis deviation, nonspecific ST-T change, and clockwise rotation.

as a bridge to myocardial recovery in response to histologyguided immunosuppressive therapy.

\section{Case Presentation}

A previously healthy, athletic 16-year-old boy (body surface area $1.82 \mathrm{~mm}^{2}$ ) was transferred to our cardiac institution with a 9-day history of a sustained high fever complicated by diarrhea and abdominal pain. He had no history of allergic disease such as bronchial asthma, or known drug allergies. He had received oral cefdinir for a few days for suspected bacterial enterocolitis two weeks previously. His symptoms had been resistant to the antibiotics and antipyretic medications given in the prior tertiary center. Upon arrival at our center, he exhibited cardiogenic shock with a body temperature of $40.5^{\circ}$, heart rate 107 beats/min (irregular), systolic pressure 90, and diastolic pressure ranging from 30 to $75 \mathrm{mmHg}$ (measured by oscillometric method, unstable), and respiratory rate $48 / \mathrm{min}$. He was mostly conscious but occasionally stuporous. No jugular distension, limb edema, or skin rash was observed. His peripheral body was cold despite his body trunk being warm. On auscultation, he had regularly irregular muffled heart sounds without a significant murmur, and some rales bibasally. The electrocardiogram revealed tachycardia, sinus rhythm, right axis deviation, nonspecific ST-T change, and clockwise rotation (Figure 1). His chest radiograph showed prominent pulmonary edema, accumulation of pleural effusion, and an enlarged cardiac shadow (Figure 2(a)). Echocardiography showed LV systolic dysfunction-LV end-diastolic volume (LVEDV) and endsystolic volume $(\mathrm{LVESV})=130 / 76 \mathrm{~mL}$; LV ejection fraction (LVEF) 42\%-a mildly thickened and echogenic LV wall, and a modest amount of pericardial effusion, without visible intraventricular thrombus (Figure 2(b)). Blood tests showed respiratory alkalosis, high concentration of markers of cardiac injury (troponin I $1843.6 \mathrm{pg} / \mathrm{mL}$ [upper limit of normal: $26.2 \mathrm{pg} / \mathrm{mL}$ ) ), heart failure (plasma brain natriuretic peptide $2671.1 \mathrm{pg} / \mathrm{mL}$ [18.4 pg/mL]), evidence of an inflammatory response (white blood cell count $8.5 \times 10^{3} / \mu \mathrm{L}$ with neutrophils $93 \%$ and eosinophils $1.5 \%$ and serum C-reactive protein $29 \mathrm{mg} / \mathrm{dL}$ ), mild hepatic failure (aspartate aminotransferase $145 \mathrm{U} / \mathrm{L}$ [38 U/L] and alanine aminotransferase $100 \mathrm{U} / \mathrm{L}[44 \mathrm{U} / \mathrm{L}])$, and almost unimpaired renal function (blood urea nitrogen $26 \mathrm{mg} / \mathrm{dL}$ [20 mg/dL] and creatinine 1.2 $\mathrm{mg} / \mathrm{dL}[1.08 \mathrm{mg} / \mathrm{dL}])$. The myeloperoxidase-anti-neutrophil cytoplasmic antibody was below the detectable range.

Despite inotropic support and fluid resuscitation, his systolic blood pressure suddenly fell to $80 \mathrm{mmHg}$ in the middle of the initial evaluation. Based on the clinical diagnosis of cardiogenic shock, an Impella 2.5 was immediately inserted into the right femoral artery via a 12-Fr sheath, followed by a right-ventricular (RV) endomyocardial biopsy (EMB) via the right internal jugular. Intact coronary arteries were subsequently confirmed by coronary angiography. Chest radiography after Impella placement showed alleviated pulmonary edema (Figure 2(c)) together with elevation of the mean arterial pressure from 51 to $72 \mathrm{mmHg}$, although the LVEF was decreased to 27\% (Figure 2(d); Supplementary Movie 1). Because of his high sustained fever, fluid resuscitation with extracellular fluid and blood products (approximately $6 \mathrm{~L} /$ day) was required to maintain the mean arterial pressure $>55 \mathrm{mmHg}$. However, the mean pulmonary wedge pressure was mildly elevated $(10-13 \mathrm{mmHg})$ without the patient displaying radiographic pulmonary edema.

Within $48 \mathrm{~h}$ after admission, eosinophilic myocarditis was diagnosed based on the histological findings (Figure 3). Immunosuppressive therapy consisting of methylprednisolone $1000 \mathrm{mg} /$ day for 3 days followed by prednisolone $1 \mathrm{mg} / \mathrm{kg} /$ day and azathioprine $2 \mathrm{mg} / \mathrm{kg} /$ day was commenced, which improved his LV systolic function in the short term (LVEF $47 \%$ by day 6) and decreased the body temperature, leading to hemodynamic stabilization. The doses of inotropes required to maintain stable hemodynamics during Impella use were decreased by day 4 (maximum dose of dobutamine was $2.2 \mu \mathrm{g} / \mathrm{kg} / \mathrm{min}$ and of dopamine 1.5 $\mu \mathrm{g} / \mathrm{kg} / \mathrm{min}$ on days 1 and 2 ).

He was gradually, day by day, weaned from the Impella, decreasing from the P8 to P2 level without showing marked end-organ failure. The Impella was surgically removed on day 7 , with his postremoval blood pressure at 120/70 $\mathrm{mmHg}$ and heart rate at 65 beats/min on with minimal inotropic support (dobutamine and dopamine, $3.0 \mu \mathrm{g} / \mathrm{kg} / \mathrm{min}$ each) to avoid sudden hemodynamic deterioration immediately after removal of the MCS. He was extubated on day 8, followed by initiation of antiheart failure medications (e.g., angiotensin-converting-enzyme inhibitor and $\beta$-blocking agent) to prevent further remodeling. The follow-up EMB, 


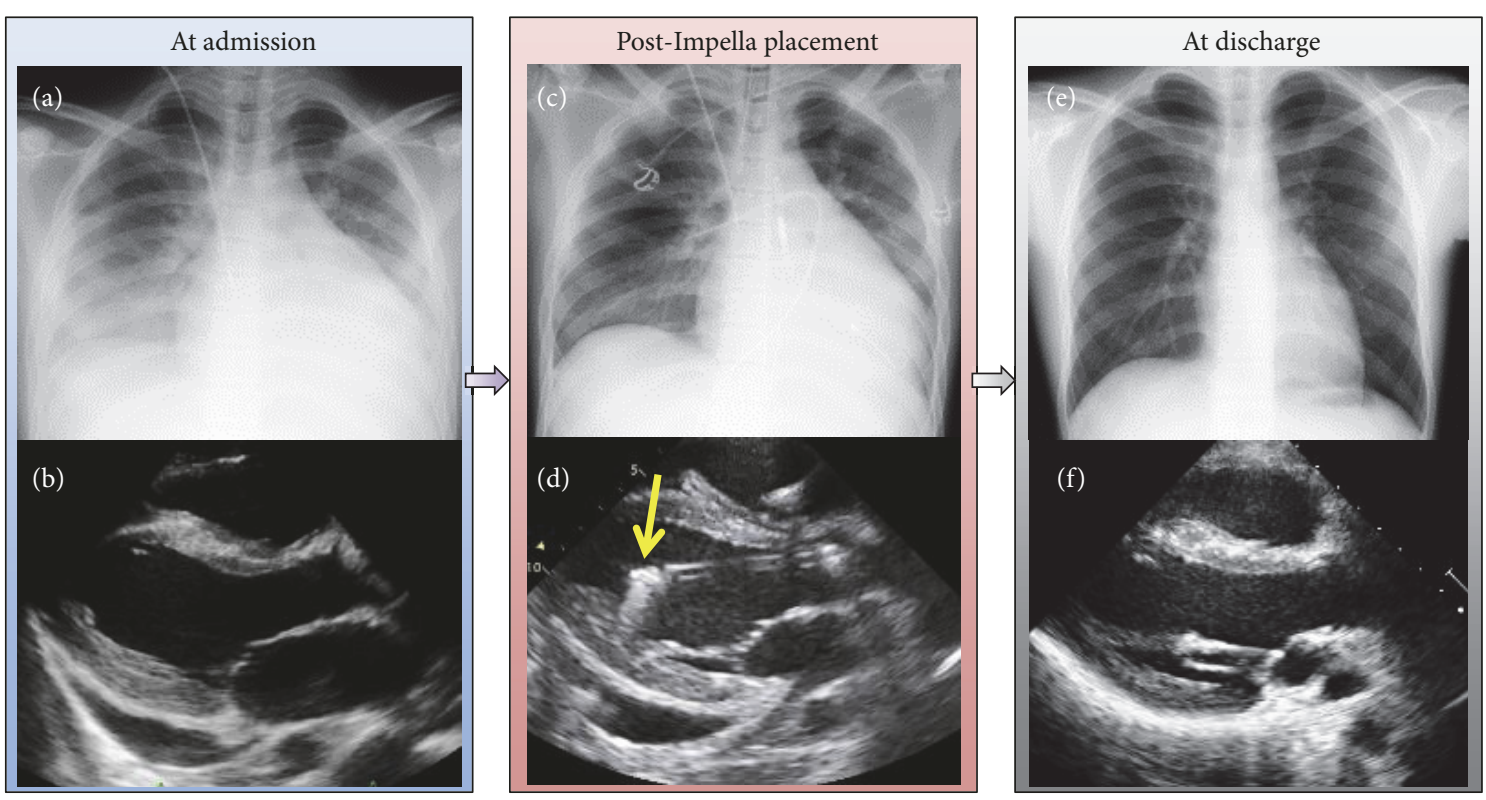

FIGURE 2: Findings on admission and after Impella placement. (a) Pre-Impella placement chest radiograph shows prominent pulmonary edema with an enlarged cardiac shadow. (b) At admission (pre-Impella placement), echocardiography, parasternal long-axis view, shows an extensively thickened left ventricle with enhanced echogenicity and a modest amount of pericardial effusion [left-ventricular ejection fraction (LVEF) 42\%]. (c) Post-Impella placement chest radiography shows less pulmonary edema. (d) Post-Impella placement echocardiography, parasternal long-axis view, shows Impella inlet parts with an acoustic shadow in the proper position (yellow arrow) (LVEF 27\%). (e) At discharge, chest radiography shows an almost normal cardiac shadow. (f) At discharge, echocardiography, parasternal long-axis view, shows improved contractility without pericardial effusion (LVEF 60\%).

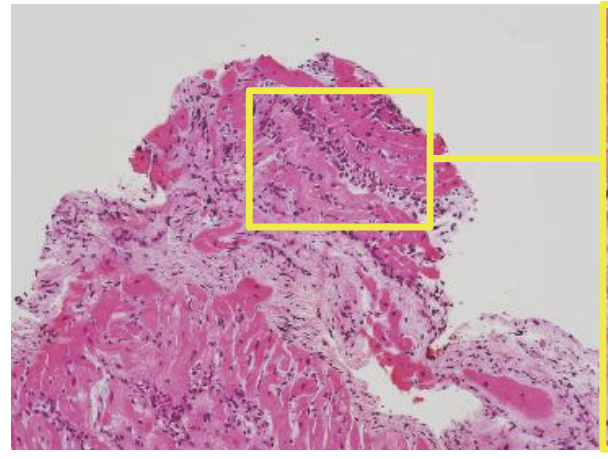

(a)

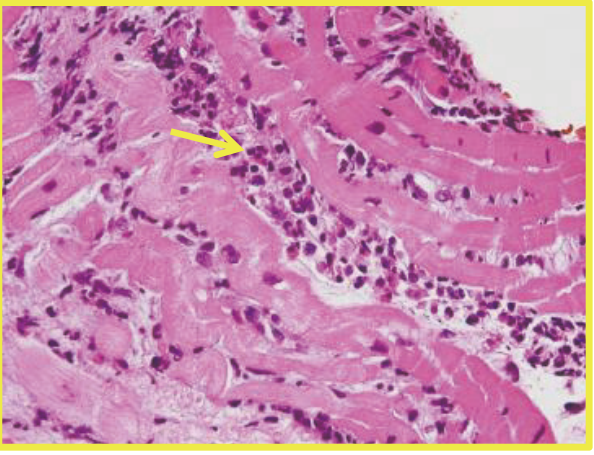

(b)

FIGURE 3: Histological findings of right-ventricular endomyocardial biopsy specimen on admission. Hematoxylin-eosin staining (formalin-fixed; paraffin-embedded) shows damaged myocardium and infiltration of degranulated eosinophils (arrow, right), lymphocytes, and neutrophils. (a) $\times 20$. (b) $\times 60$.

performed 2 weeks after his admission, revealed improved infiltration of eosinophils and lymphocytes and some degree of fibrosis (Supplementary Figure 1). Azathioprine was tapered off, whereas prednisolone dose was gradually tapered to $30 \mathrm{mg} /$ day. He was discharged from hospital 6 weeks after the admission without a major complication (Figure 4).

Thallium-201 and iodine (I)-123 $\beta$-methyl-p-iodophenylpentadecanoic acid (BMIPP) dual-tracer myocardial scintigraphy 3 weeks after admission showed a patchy deficit of perfusion at the anterolateral wall and a discordantly larger deficit of fatty acid metabolism (perfusion-metabolism mismatch), suggesting regional myocardial damage with potential reversibility (Figure 5).

Cardiac 1.5-T magnetic resonance imaging (CMR) 4 weeks after admission showed no high-intensity areas on T2weighted imaging or late-gadolinium enhancement (LGE), but prolonged native T1 mapping at papillary muscle level, indicating myocardial edema (Figure 6; Supplementary Movie 2).

At discharge from the hospital, follow-up chest radiography revealed diminishing cardiomegaly with no pulmonary congestion (Figure 2(e)). At the same time echocardiography showed recovery of contractility with LVEF $60 \%$ 

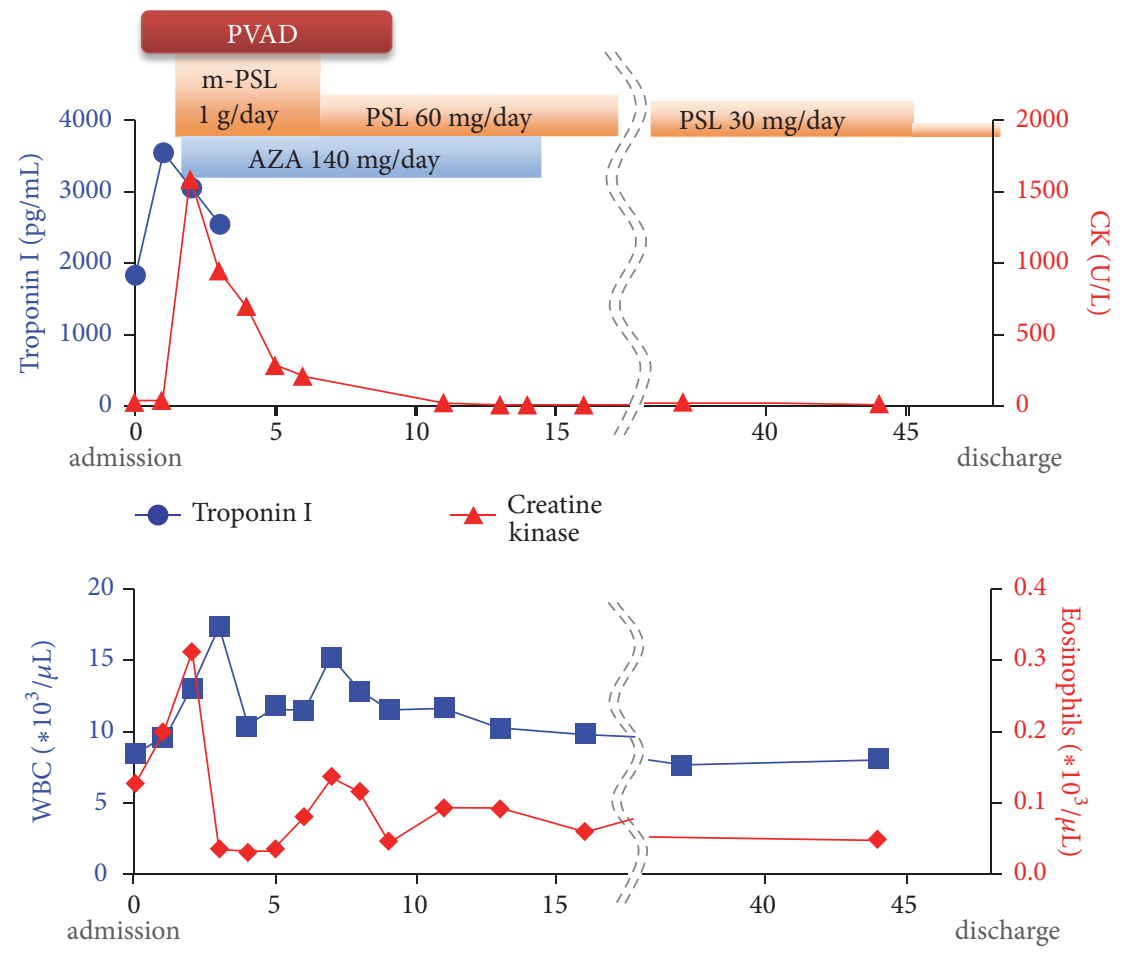

$-\begin{aligned} & \text { White blood } \\ & \text { cells count } \\ & \text { count }\end{aligned}$
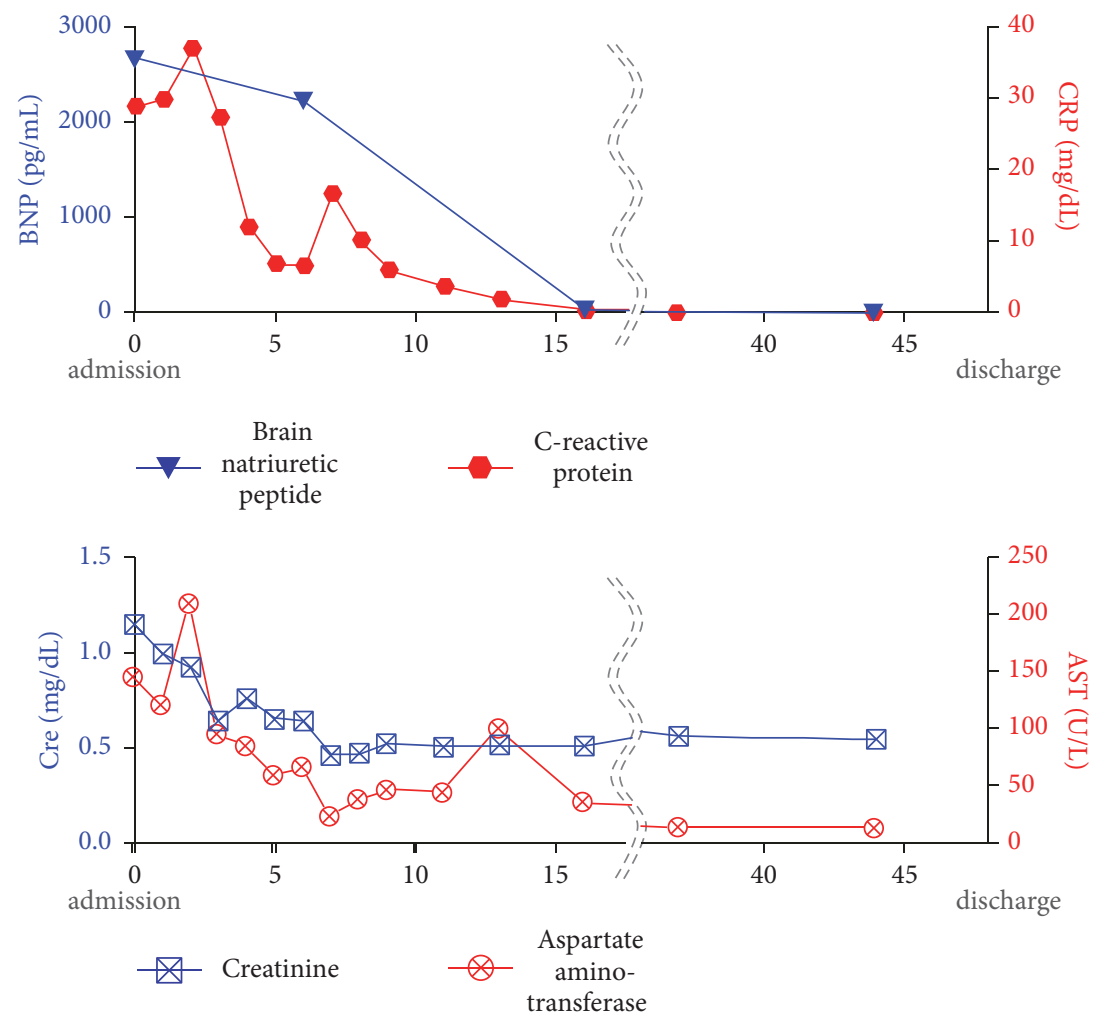

FIGURE 4: Changes in blood markers during hospitalization. Graphs showing changes in serum troponin I and creatine-kinase concentrations, white blood cell and eosinophil counts, plasma brain natriuretic peptide, serum C-reactive protein, serum creatinine, and serum aspartate amino transferase concentrations, together timing of with mechanical circulatory support and immunosuppressive therapy. PVAD, percutaneous ventricular assist device; m-PSL, methyl-prednisolone; PSL, prednisolone. 


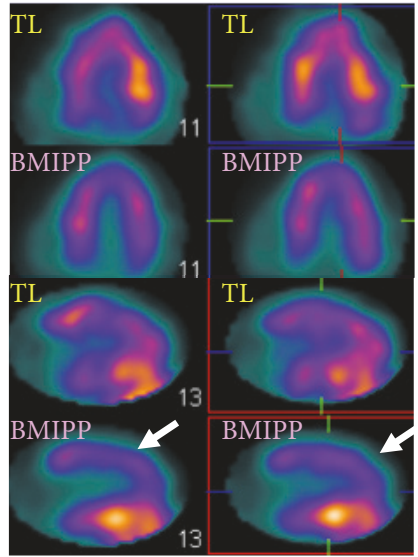

(a) long axis

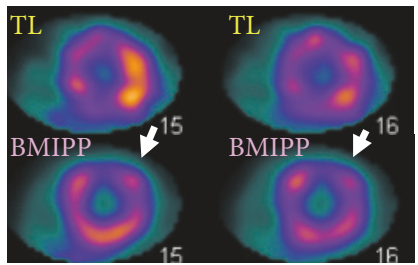

(b) short axis

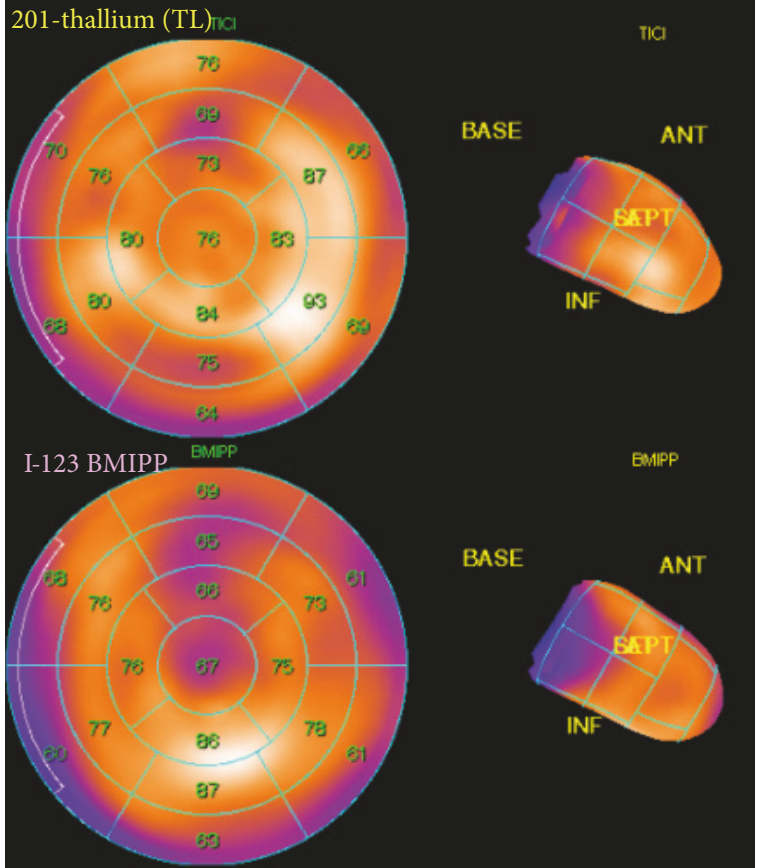

(c) polar map

FIGURE 5: Thallium-201 and iodine-123 $\beta$-methyl-p-iodophenylpentadecanoic acid dual-tracer myocardial scintigraphy 3 weeks after admission. Slight patchy deficit was found for myocardial thallium uptake in the anterolateral wall and further decreased uptake of BMIPP, which was consistent with a mild perfusion-metabolism mismatch (white arrows). (a) Long-axis 2D view. (b) Short axis 2D view. (c) Polar map. 2D, two-dimensional; ANT, anterior; INF, inferior; TL, thallium.

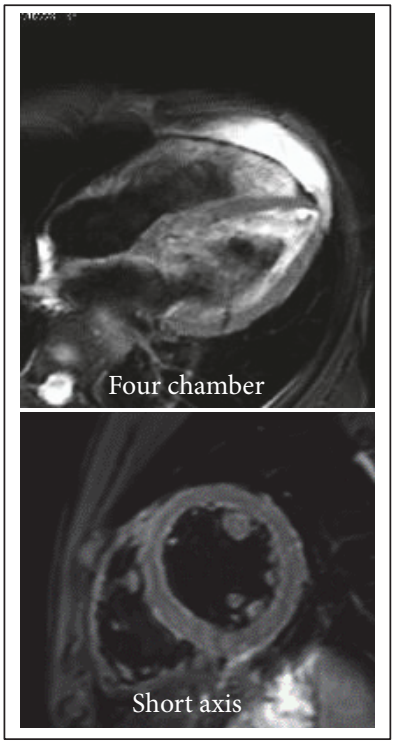

(a) Black-blood T2

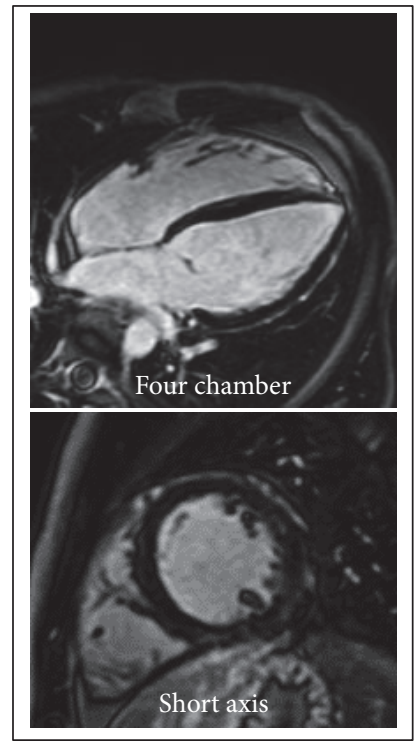

(b) Late-gadolinium enhancement

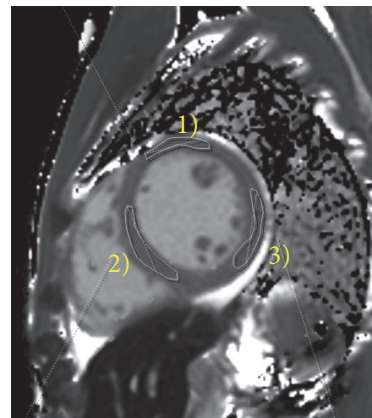

(c) Native T1 mapping

FIGURE 6: Cardiac 1.5-T magnetic resonance imaging 4 weeks after admission. (a) T2-weighted black-blood images show no significant highintensity signal. (b) Late-gadolinium enhancement shows no significant focal high-intensity signal. (c) Native T1 mapping analysis shows mildly elevated values: (1) anterior wall, $1193.01 \pm 36.34 \mathrm{msec}$, (2) septal wall, $1193.05 \pm 32.03 \mathrm{msec}$, and (3) posterolateral wall, $1198.87 \pm 42.86$ msec. 
(Figure 2(f)). The patient was weaned off steroid therapy 5 months after the onset of his myocarditis and showed no evidence of recurrence in the subsequent 7 months.

\section{Discussion}

At the time of presentation to our hospital, we recognized that our patient had complicated acute myocarditis (LVEF <50\%), which is associated with a worse prognosis and therefore important to identify on presentation [13]. The patient fully recovered from fulminant EM owing to the bridging MCS treatment with PVAD, combined with immunosuppressive therapy based on the histological findings of immediate EMB. Although his cardiac function was temporarily severely impaired, the patient showed no major end-organ dysfunction, severe pulmonary edema, or irreversible myocardial damage. These favorable outcomes may be attributed to the immediate use of PVAD, which supported antegrade flow and unloaded the LV preload.

\subsection{Diagnosis and Medical Treatment of Eosinophilic} Myocarditis. The proposed underlying etiologies of EM include hypersensitivity reactions $[6,14]$, autoimmunemediated disease [e.g., eosinophilic granulomatosis with polyangiitis $[15,16]$; hypereosinophilic syndrome or its variants [17-19]], parasitic infections [20], and cancer [19]. However, the cause in a substantial proportion of patients remains unknown, as in our case. Our patient had no history of allergic disease, including bronchial asthma or drug allergy, and the fact that he was negative for myeloperoxidase-anti-neutrophil cytoplasmic antibody makes eosinophilic granulomatosis with polyangiitis unlikely. He had received oral cefdinir; however, it had been ceased a week prior to development of cardiac symptoms. Preceding enterocolitis (viral infection) or oral antibiotics are potential underlying causes for the development of EM; however, the precise causality cannot be determined. In fulminant myocarditis, acute (usually within 2 weeks of symptom onset) worsening of the patient's hemodynamics leads to cardiogenic shock [21-23]. Thus, the clinical course of fulminant EM is often fatal, with in-hospital mortality at approximately one-third or one-half of these patients $[5,6,24]$.

Peripheral eosinophilia may be absent in $25 \%$ of patients [5], as was the case with our patient. Eosinophilic cationic protein [25] and total immunoglobulin E may play roles in the pathogenesis of EM; however, we did not measure these markers. Only EMB allows a definitive diagnosis of EM [2628]. The European Society of Cardiology Working Group proposed using EMB as a tool for monitoring and guiding therapy in patients with specific forms of myocarditis, such as EM, that potentially could be treated with immunosuppression [29]. Although no immunosuppressive therapy protocol for fulminant EM has yet been established, previous reports have suggested that steroids are effective (i.e., methylprednisolone $1000 \mathrm{mg} /$ day for 3 days followed by prednisolone $(1 \mathrm{mg} / \mathrm{kg} /$ day $)[30,31]$ or azathioprine $(2 \mathrm{mg} / \mathrm{kg} /$ day $)$ in combination with steroids is effective [32]). These reports on immunosuppressive therapy are consistent with our case in which the patient exhibited an immediate favorable clinical and histological response to the immunosuppressive therapy. Patients with EM may show a nontypical, undefined LGE pattern [5]. We did not perform CMR imaging in the acute phase because the patient needed to rest. A CMR performed 4 weeks later revealed no LGE; we speculate that early initiation of immunosuppressive therapy prevented prominent irreversible fibrotic change.

3.2. Mechanical Circulatory Support for Cardiogenic Shock Including Fulminant Myocarditis. The use of VA-ECMO is an attractive MCS option for patients in severe cardiogenic shock [7-10]. However, disadvantageous effects associated with the lack of LV unloading (e.g., increased LV filling, elevated pulmonary capillary pressures, or reduced subendocardial myocardial coronary flow) are of great concern $[8,33]$.

The Impella 2.5 is a percutaneously inserted device equipped with a miniature, nonpulsatile, axial-flow Archimedes-screw pump that propels it from the left ventricle to the ascending aorta. By unloading the left ventricle and supporting flow, it reduces the total mechanical work, subendocardial ischemia, myocardial oxygen demand, pulmonary capillary wedge pressure, and end-organ malperfusion $[8,34-40]$. A durable ventricular assist device is another choice of MCS to unload the left ventricle, but use of this device in patients with fulminant myocarditis is associated with a lower survival rate than percutaneous $\mathrm{V}$-A ECMO, potentially associated with its lack of rapidity to ameliorate hemodynamic instability $[41,42]$. Importantly, before insertion of Impella, the presence of intraventricular thrombus should be carefully ruled out to avoid a procedurerelated embolism. Such a step is particularly important in patients with EM or those who have experienced apical or extensive myocardial infarction as these patients frequently harbor a mural or apical thrombus. Careful investigation of LV size is also important, as patients with acute myocarditis frequently have a small LV cavity because of myocardial edema; this may lead to suboptimal positioning of the tip of the catheter.

Several previous publications have reported successful results in patients with fulminant myocarditis using the LV Impella, including a 13-year-old boy without a histological diagnosis (Impella 5.0, bridge-to-recovery) [40], a 44year-old woman with giant cell myocarditis (Impella 2.5, bridge-to-durable LV device) [43], and a 49-year-old woman with suspected autoimmune etiology (Impella CP, bridge-torecovery) [44]. In our case, continuous fluid resuscitation had been required even after the PVAD insertion as the patient had revealed high sustained fever before immunosuppressive therapy exerted its effect. A combination of "mixed" cardiogenic and circulatory shock can occur in patients with a low cardiac output and an acute inflammatory response; the left-ventricular unloading provided by PVAD was advantageous in refilling intraventricular volume without resulting in development of pulmonary edema.

Our case is specific in that the EMB-driven histological diagnosis played a crucial role in initiating early immunosuppressive therapy, which led to immediately improved myocardial function, allowing us to wean the patient off the 
Impella. The CMR findings in our case indicated increased extracellular volume (elevated T1 mapping values) but not apparent myocardial fibrosis, as represented by the LGE [45], also suggesting the effectiveness of the bridging MCS strategy in limiting the extent of irreversible myocardial damage.

Combinations of the LV PVAD with other MCS may be effective in various clinical scenarios. For instance, patients with cardiogenic shock accompanied by severely impaired oxygenation may benefit from a combination of LV PVAD and V-A ECMO, by oxygenating and flow-supporting while unloading the left ventricle [46-48]. Another clinical scenario is prolonged cardiogenic shock with RV dysfunction, which is an under-recognized predictor of a poor prognosis [34]. Patients with fulminant/acute myocarditis with severe biventricular failure may benefit from MCS using the LV and RV PVAD [49].

\section{Conclusion}

For fulminant EM, the short-term use of PVAD, together with immunosuppressive therapy guided by an immediate histological investigation, may be an effective bridging strategy to myocardial recovery.

\section{Conflicts of Interest}

The authors declare that they have no conflicts of interest.

\section{Supplementary Materials}

The supplemental materials are movies to demonstrate that the heart function was depressed. Supplementary Figure 1: histological findings of the right-ventricular endomyocardial biopsy specimen 2 weeks after admission. (Supplementary Materials)

\section{References}

[1] A. M. Al Ali, L. P. Straatman, M. F. Allard, and A. P. Ignaszewski, "Eosinophilic myocarditis: case series and review of literature," Canadian Journal of Cardiology, vol. 22, no. 14, pp. 1233-1237, 2006.

[2] T. Fozing, N. Zouri, A. Tost et al., "Management of a patient with eosinophilic myocarditis and normal peripheral eosinophil count," Circulation: Heart Failure, vol. 7, no. 4, pp. 692-694, 2014.

[3] A. Pollack, A. R. Kontorovich, V. Fuster, and G. W. Dec, "Viral myocarditis-diagnosis, treatment options, and current controversies," Nature Reviews Cardiology, vol. 12, no. 11, pp. 670-680, 2015.

[4] I. Kindermann, C. Barth, F. Mahfoud et al., "Update on myocarditis," Journal of the American College of Cardiology, vol. 59, no. 9, pp. 779-792, 2012.

[5] M. Brambatti, M. V. Matassini, E. D. Adler, K. Klingel, P. G. Camici, and E. Ammirati, "Eosinophilic myocarditis," Journal of the American College of Cardiology, vol. 70, no. 19, pp. 23632375, 2017.

[6] G. P. Bourgeois, J. A. Cafardi, V. Groysman, and L. C. Hughey, "A review of DRESS-associated myocarditis," Journal of the
American Academy of Dermatology, vol. 66, no. 6, pp. e229e236, 2012.

[7] K. Werdan, S. Gielen, H. Ebelt, and J. S. Hochman, "Mechanical circulatory support in cardiogenic shock," European Heart Journal, vol. 35, no. 3, pp. 156-167, 2014.

[8] C. Tschöpe, S. Van Linthout, O. Klein et al., "Mechanical unloading by fulminant myocarditis: LV-IMPELLA, ECMELLA, BI-PELLA, and PROPELLA Concepts," Journal of Cardiovascular Translational Research, 2018.

[9] P.-S. Hsu, J.-L. Chen, G.-J. Hong et al., "Extracorporeal membrane oxygenation for refractory cardiogenic shock after cardiac surgery: predictors of early mortality and outcome from 51 adult patients," European Journal of Cardio-Thoracic Surgery, vol. 37, no. 2, pp. 328-333, 2010.

[10] J. W. Diddle, M. C. Almodovar, S. K. Rajagopal, P. T. Rycus, and R. R. Thiagarajan, "Extracorporeal membrane oxygenation for the support of adults with acute myocarditis," Critical Care Medicine, vol. 43, no. 5, pp. 1016-1025, 2015.

[11] M. L. Paden, S. A. Conrad, P. T. Rycus, and R. R. Thiagarajan, "Extracorporeal life support organization registry report 2012," ASAIO Journal, vol. 59, no. 3, pp. 202-210, 2013.

[12] S. S. Basra, P. Loyalka, and B. Kar, "Current status of percutaneous ventricular assist devices for cardiogenic shock," Current Opinion in Cardiology, vol. 26, no. 6, pp. 548-554, 2011.

[13] E. Ammirati, M. Cipriani, C. Moro et al., "Clinical presentation and outcome in a contemporary cohort of patients with acute myocarditis," Circulation, vol. 138, no. 11, pp. 1088-1099, 2018.

[14] M. Pieroni, R. Cavallaro, C. Chimenti, E. Smeraldi, and A. Frustaci, "Clozapine-induced hypersensitivity myocarditis," Chest, vol. 126, no. 5, pp. 1703-1705, 2004.

[15] A. T. Masi, G. G. Hunder, J. T. Lie et al., "The American College of Rheumatology 1990 criteria for the classification of churg-strauss syndrome (allergic granulomatosis and angiitis)," Arthritis \& Rheumatism, vol. 33, no. 8, pp. 1094-1100, 1990.

[16] J. C. Jennette, R. J. Falk, P. A. Bacon et al., "2012 revised international Chapel Hill consensus conference nomenclature of vasculitides," Arthritis \& Rheumatology, vol. 65, no. 1, pp. 111, 2013.

[17] M. H. Roehrl, M. P. Alexander, S. B. Hammond, M. Ruzinova, J. Y. Wang, and C. J. O'Hara, "Eosinophilic myocarditis in hypereosinophilic syndrome," American Journal of Hematology, vol. 86, no. 7, pp. 607-608, 2011.

[18] P. Valent, A. D. Klion, H.-P. Horny et al., "Contemporary consensus proposal on criteria and classification of eosinophilic disorders and related syndromes," The Journal of Allergy and Clinical Immunology, vol. 130, no. 3, pp. 607-612.e9, 2012.

[19] E. Ammirati, M. Stucchi, M. Brambatti et al., "Eosinophilic myocarditis: a paraneoplastic event," The Lancet, vol. 385, no. 9986, p. 2546, 2015.

[20] K. Enko, T. Tada, K. O. Ohgo et al., "Fulminant eosinophilic myocarditis associated with visceral larva migrans caused by toxocara canis infection," Circulation Journal, vol. 73, no. 7, pp. 1344-1348, 2009.

[21] S. Gupta, D. W. Markham, M. H. Drazner, and P. P. Mammen, "Fulminant myocarditis," Nature Clinical Practice Cardiovascular Medicine, vol. 5, no. 11, pp. 693-706, 2008.

[22] R. E. McCarthy III, J. P. Boehmer, R. H. Hruban et al., "Longterm outcome of fulminant myocarditis as compared with acute (nonfulminant) myocarditis," The New England Journal of Medicine, vol. 342, no. 10, pp. 690-695, 2000. 
[23] M. Matsumoto, Y. Asaumi, Y. Nakamura et al., "Clinical determinants of successful weaning from extracorporeal membrane oxygenation in patients with fulminant myocarditis," ESC Heart Failure, vol. 5, no. 4, pp. 675-684, 2018.

[24] E. Ammirati, M. Cipriani, M. Lilliu et al., "Survival and left ventricular function changes in fulminant versus nonfulminant acute myocarditis," Circulation, vol. 136, no. 6, pp. 529-545, 2017.

[25] S. Kawano, J. Kato, N. Kawano et al., "Clinical features and outcomes of eosinophilic myocarditis patients treated with prednisolone at a single institution over a 27-year period," Internal Medicine, vol. 50, no. 9, pp. 975-981, 2011.

[26] U. Baandrup, "Eosinophilic myocarditis," Herz, vol. 37, no. 8, pp. 849-853, 2012.

[27] F. Ginsberg and J. E. Parrillo, "Eosinophilic myocarditis," Heart Failure Clinics, vol. 1, no. 3, pp. 419-429, 2005.

[28] A. L. P. Caforio, S. Pankuweit, E. Arbustini et al., "Current state of knowledge on aetiology, diagnosis, management, and therapy of myocarditis: a position statement of the European Society of Cardiology Working Group on Myocardial and Pericardial Diseases," European Heart Journal, vol. 34, no. 33, pp. 26362648, 2013.

[29] E. Ammirati, G. Veronese, M. Cipriani et al., "Acute and fulminant myocarditis: a pragmatic clinical approach to diagnosis and treatment," Current Cardiology Reports, vol. 20, no. 11, p. 114, 2018.

[30] D. Corradi, A. Vaglio, R. Maestri et al., "Eosinophilic myocarditis in a patient with idiopathic hypereosinophilic syndrome: Insights into mechanisms of myocardial cell death," Human Pathology, vol. 35, no. 9, pp. 1160-1163, 2004.

[31] L. Galiuto, M. Enriquez-Sarano, S. R. Guy et al., "Eosinophilic myocarditis manifesting as myocardial infarction: early diagnosis and successful treatment," Mayo Clinic Proceedings, vol. 72, no. 7, pp. 603-610, 1997.

[32] A. Aggarwal, P. Bergin, P. Jessup, and D. Kaye, "Hypersensitivity myocarditis presenting as cardiogenic shock," The Journal of Heart and Lung Transplantation, vol. 20, no. 11, pp. 1241-1244, 2001.

[33] A. Barbone, P. G. Malvindi, P. Ferrara, and G. Tarelli, "Left ventricle unloading by percutaneous pigtail during extracorporeal membrane oxygenation," Interactive CardioVascular and Thoracic Surgery, vol. 13, no. 3, pp. 293-295, 2011.

[34] M. Seyfarth, D. Sibbing, I. Bauer et al., "A randomized clinical trial to evaluate the safety and efficacy of a percutaneous left ventricular assist device versus intra-aortic balloon pumping for treatment of cardiogenic shock caused by myocardial infarction," Journal of the American College of Cardiology, vol. 52, no. 19, pp. 1584-1588, 2008.

[35] L. D. Sauren, R. E. Accord, K. Hamzeh et al., "Combined impella and intra-aortic balloon pump support to improve both ventricular unloading and coronary blood flow for myocardial recovery: an experimental study," Artificial Organs, vol. 31, no. 11, pp. 839-842, 2007.

[36] B. Meyns, J. Stolinski, V. Leunens, E. Verbeken, and W. Flameng, "Left ventricular support by Catheter-Mountedaxial flow pump reduces infarct size," Journal of the American College of Cardiology, vol. 41, no. 7, pp. 1087-1095, 2003.

[37] M. Remmelink, K. D. Sjauw, J. P. Henriques et al., "Effects of left ventricular unloading by impella recover LP2.5 on coronary hemodynamics," Catheterization and Cardiovascular Interventions, vol. 70, no. 4, pp. 532-537, 2007.

[38] R. A. Aqel, F. G. Hage, and A. E. Iskandrian, "Improvement of myocardial perfusion with a percutaneously inserted left ventricular assist device," Journal of Nuclear Cardiology, vol. 17, no. 1, pp. 158-160, 2010.

[39] K. Lam, K. D. Sjauw, J. P. Henriques, C. Ince, and B. A. de Mol, "Improved microcirculation in patients with an acute STelevation myocardial infarction treated with the Impella LP2.5 percutaneous left ventricular assist device," Clinical Research in Cardiology, vol. 98, no. 5, pp. 311-318, 2009.

[40] J. G. Andrade, H. Al-Saloos, A. Jeewa, G. G. S. Sandor, and A. Cheung, "Facilitated cardiac recovery in fulminant myocarditis: pediatric use of the Impella LP 5.0 pump," The Journal of Heart and Lung Transplantation, vol. 29, no. 1, pp. 96-97, 2010.

[41] Y. Asaumi, S. Yasuda, I. Morii et al., "Favourable clinical outcome in patients with cardiogenic shock due to fulminant myocarditis supported by percutaneous extracorporeal membrane oxygenation," European Heart Journal, vol. 26, no. 20, pp. 2185-2192, 2005.

[42] M. A. Acker, "Mechanical circulatory support for patients with acute-fulminant myocarditis," The Annals of Thoracic Surgery, vol. 71, no. 3, pp. S73-S76, 2001.

[43] H. Suradi and J. A. Breall, "Successful use of the impella device in giant cell Myocarditis as a bridge to permanent left Ventricular mechanical support," Texas Heart Institute Journal, vol. 38, no. 4, pp. 437-440, 2011.

[44] H. Fox, M. Farr, D. Horstkotte, and C. Flottmann, "Fulminant myocarditis managed by extracorporeal life support (Impella ${ }^{\circledR}$ CP): a rare case," Case Reports in Cardiology, vol. 2017, Article ID 9231959, 4 pages, 2017.

[45] V. M. Ferreira, S. K. Piechnik, E. Dall'Armellina et al., "Native T1-mapping detects the location, extent and patterns of acute myocarditis without the need for gadolinium contrast agents," Journal of Cardiovascular Magnetic Resonance, vol. 16, no. 1, p. 36, 2014.

[46] S. Narain, G. Paparcuri, T. M. Fuhrman et al., "Novel combination of impella and extra corporeal membrane oxygenation as a bridge to full recovery in fulminant myocarditis," Case Reports in Critical Care, vol. 2012, Article ID 459296, 3 pages, 2012.

[47] S. V. Chaparro, A. Badheka, G. R. Marzouka et al., "Combined use of impella left ventricular assist device and extracorporeal membrane oxygenation as a bridge to recovery in fulminant myocarditis," ASAIO Journal, vol. 58, no. 3, pp. 285-287, 2012.

[48] S. Beurtheret, P. Mordant, X. Paoletti et al., "Emergency circulatory support in refractory cardiogenic shock patients in remote institutions: a pilot study (the cardiac-RESCUE program)," European Heart Journal, vol. 34, no. 2, pp. 112-120, 2013.

[49] F. Pappalardo, A. M. Scandroglio, and A. Latib, "Full percutaneous biventricular support with two Impella pumps: the BiPella approach," ESC Heart Failure, vol. 5, no. 3, pp. 368-371, 2018. 


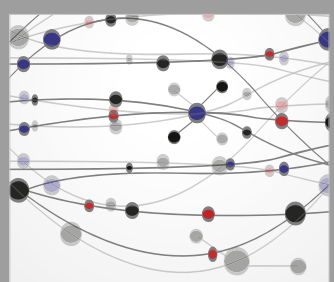

The Scientific World Journal
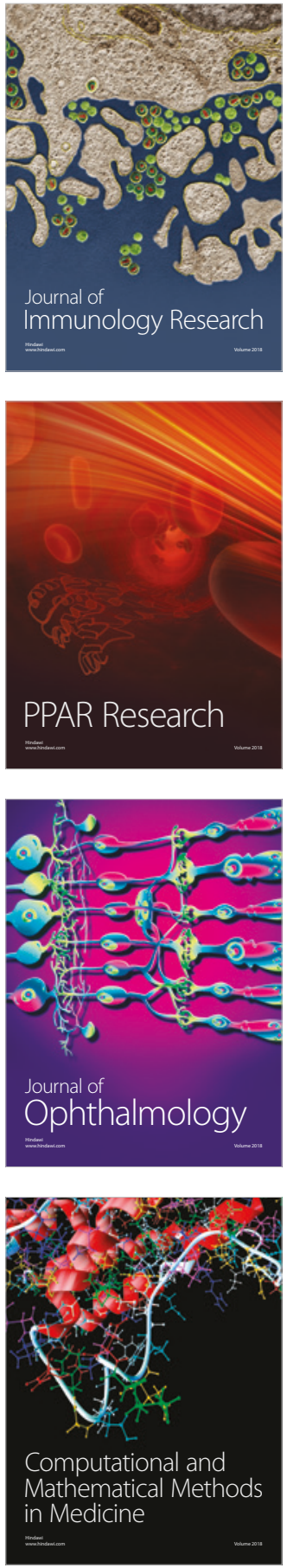

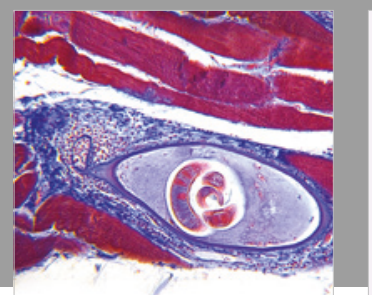

Gastroenterology Research and Practice

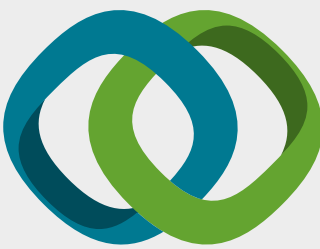

\section{Hindawi}

Submit your manuscripts at

www.hindawi.com
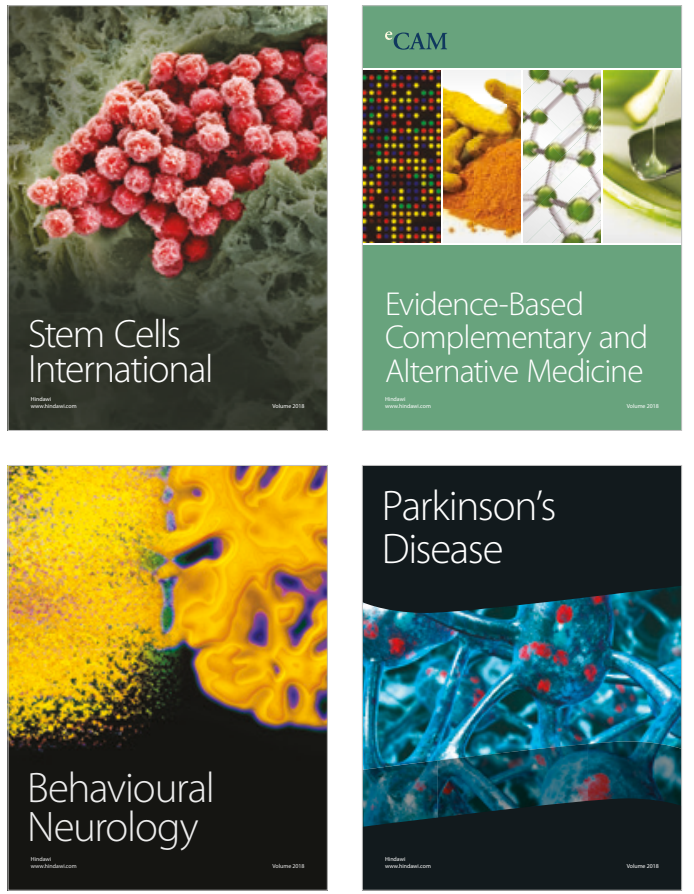

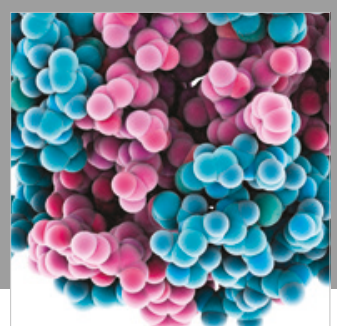

ournal of

Diabetes Research

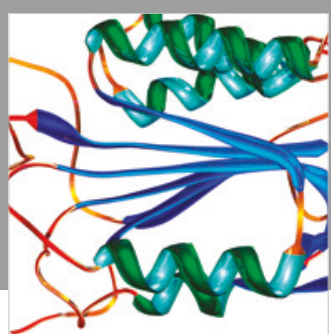

Disease Markers
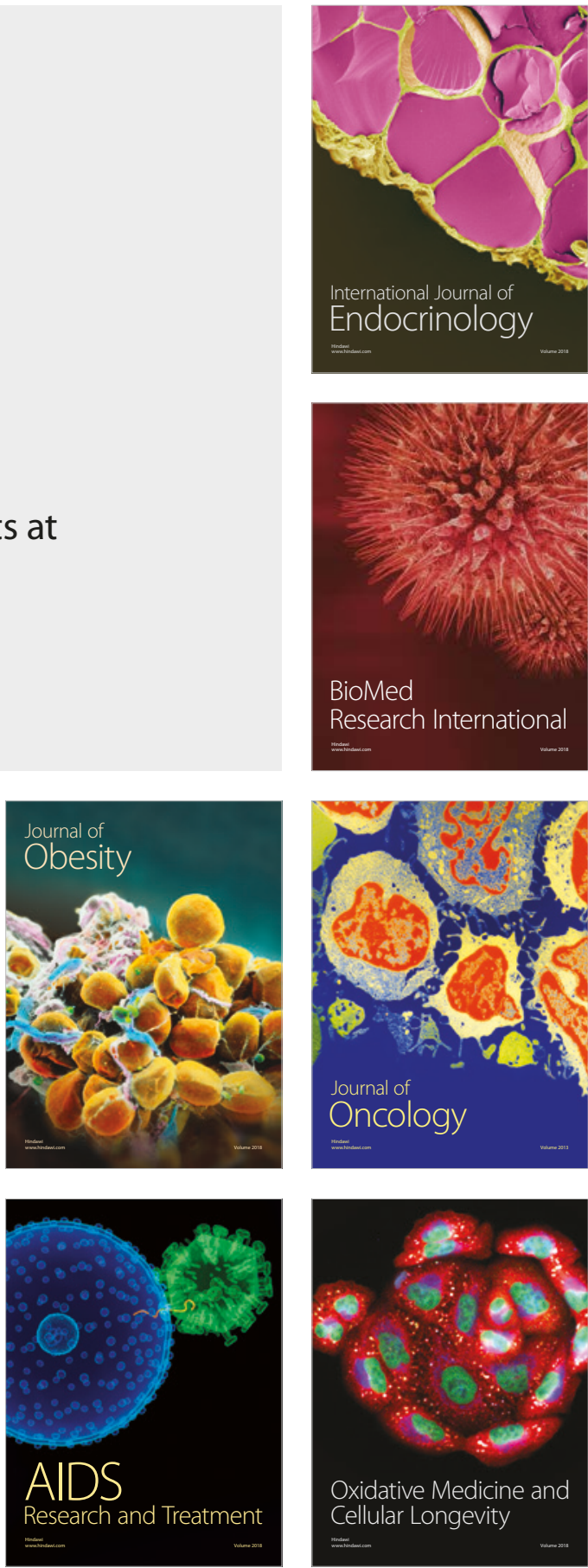